\title{
Investigating the Susceptibility of Treatment- Resistant Oesophageal Tumours to Natural Killer Cell-Mediated Responses.
}

\section{Eimear Mylod}

Trinity College: The University of Dublin Trinity College

\section{Ellen Mckenna}

Trinity College: The University of Dublin Trinity College

\section{Maria Davern}

Trinity College: The University of Dublin Trinity College

\section{Martin P Barr}

Trinity College: The University of Dublin Trinity College

\section{Noel E Donlon}

Trinity College Dublin: The University of Dublin Trinity College

\section{Becky AS Bibby}

University of Manchester Institute of Science and Technology Department of Biomolecular Science: The University of Manchester Faculty of Biology Medicine and Health

\section{Anshul Bhardwaj}

Trinity College: The University of Dublin Trinity College

John V Reynolds

Trinity College Dublin: The University of Dublin Trinity College

\section{Joanne Lysaght}

Trinity College: The University of Dublin Trinity College

\section{Stephen G. Maher}

Trinity College: The University of Dublin Trinity College

\section{Melissa J. Conroy ( $\square$ meconroy@tcd.ie)}

Trinity College: The University of Dublin Trinity College https://orcid.org/0000-0002-3822-0442

\section{Research Article}

Keywords: NK cells, oesophageal adenocarcinoma, NK cell receptors, treatment-resistance, immunotherapy.

Posted Date: February 23rd, 2022

DOI: https://doi.org/10.21203/rs.3.rs-931913/v1 
License: (c) (i) This work is licensed under a Creative Commons Attribution 4.0 International License. Read Full License

Version of Record: A version of this preprint was published at Clinical and Experimental Medicine on April 1st, 2022. See the published version at https://doi.org/10.1007/s10238-022-00811-6. 


\section{Abstract}

Introduction: The majority of oesophageal adenocarcinoma (OAC) patients do not respond to multimodal treatment regimens and face dismal survival rates. Natural killer (NK) cells are crucial anti-tumour immune cells, and this study investigated the susceptibility of treatment-resistant OAC cells to these potent tumour killers.

Methods: Natural killer receptor (NKR) ligand expression by OE33CisP (cisplatin-sensitive) and OE33CisR (cisplatin-resistant) cells was investigated. The immunomodulatory effects of OE33CisP and OE33CisR cells on NK cell phenotype and function were assessed. Finally, the impact of chemotherapy regimens on NKR ligand shedding was examined.

Results: Our data revealed significantly less surface expression of activating ligands B7-H6, MICA/B, ULBP-3 and activating/inhibitory ligands PVRL-1 and PVRL-4 by OE33CisR cells, compared to OE33CisP cells. Co-culture with OE33CisR cells reduced the frequencies of $\mathrm{Nkp}^{+} \mathrm{O}^{+}$and $\mathrm{NKp} 46^{+} \mathrm{NK}$ cells and increased frequencies of $\mathrm{TIGIT}^{+}, \mathrm{FasL}^{+}$and $\mathrm{TRAIL}^{+} \mathrm{NK}$ cells. Frequencies of IFN- $\gamma$-producing NK cells increased while frequencies of TIM- $3^{+}$NK cells decreased after culture with OE33CisP and OE33CisR cells. Frequencies of circulating NKP30 ${ }^{+}$NK cells were significantly lower in OAC patients with the poorest treatment response and in patients who received FLOT chemotherapy. B7-H6 shedding by OAC tumour cells was induced by FLOT.

Conclusion: OE33CisR cells express less activating NKR ligands than OE33CisP cells and have differential effects on NKR expression by NK cells. However, neither cell line significantly dampened NK cell cytokine production, death receptor expression or degranulation. Finally, our data indicate that FLOT chemotherapy and obesity may promote B7-H6 shedding and immune evasion with detrimental consequences in OAC patients.

\section{Introduction}

Oesophageal adenocarcinoma (OAC) is an aggressive malignancy with a dismal 5-year survival rate of $\sim 19 \%$, placing it as the sixth highest cause of cancer-related death globally [1]. Late presentation of disease and low treatment response rates in OAC greatly contribute to its poor prognosis. For locally advanced OAC, the current standard-of-care is chemo-radiotherapy (CRT) and surgical resection, however, only $\sim 30 \%$ of these patients have a complete response, meaning that $\sim 70 \%$ are resistant to standard-ofcare therapies [2].

As immunotherapies begin to change the landscape of OAC, there is space for cellular based immunotherapies to significantly contribute to this field. With the recent approval of immune checkpoint inhibitor nivolumab plus chemotherapy as a first line treatment for advanced gastric cancer, it is evident that immunotherapies are beginning to make an impact in this space [3]. However, immune checkpoint inhibitor efficacy in gastric cancer stands at $22 \%$, meaning there is a large population of patients who do 
not gain clinical benefit from currently available immunotherapies [4]. As such, a large population of patients may still be exposed to standard-of-care chemotherapies, such as cisplatin, as a first-line treatment.

Natural Killer (NK) cells are innate lymphocytes that play a critical role in tumour eradication and it is wellestablished that their dysfunction in cancer is associated with poorer patient outcomes [5]. Activated NK cells are potent cytokine producers and elicit powerful cytotoxic activities to induce tumour cell death via perforin/granzyme-mediated-mechanisms, Fas ligand (FasL) and TNF-related apoptosis-inducing ligand (TRAIL) [6-8]. Unlike T cells, NK cells do not require specific antigen recognition via the major histocompatibility complex (MHC) and instead become activated through a fine balance of ligation with their inhibitory and activating receptors [9]. NK cell receptors (NKRs) include the NK group 2 (NKG2) family of receptors, such as the NKG2A inhibitory and NKG2D activating receptors and the activating natural cytotoxicity receptors (NCR), natural killer cell p30-related protein (NKp30) and NKp46 [9].

Adoptive T cell-based therapies have been at the fore of cellular immunotherapies for cancer and have been FDA-approved for multiple blood malignancies [10]. However, T cells have a number of perceived disadvantages compared to their innate lymphocyte counterparts in NK cells, including the requirement for antigen specificity and the requirement to be sourced autologously [11]. Indeed, this limits their applicability and scalability, precluding them from becoming a truly off-the-shelf immunotherapeutic option [11]. As such, NK cells present themselves as an adept alternative to T cells as a mediator of cellular based immunotherapies and have been explored extensively [12].

To our knowledge, we have characterised for the first time, the NKR ligand profiles and immunomodulatory properties of chemotherapy-resistant and chemotherapy-sensitive OAC tumour cells. Importantly, we have examined the susceptibility of chemotherapy-resistant OAC tumours to NK cellmediated responses and provide novel insights into the amenability of NK cell therapies for a patient cohort of whom $70 \%$ derive no clinical benefit from current treatment modalities.

\section{Materials And Methods}

\section{Cell Culture}

The OE33 oesophageal adenocarcinoma cell line was purchased from the European collection of cell cultures. Cisplatin-sensitive (OE33CisP) and cisplatin-resistant (OE33CisR) OE33 cells were derived from the original parent OE33 cell line as previously described $[13,14]$. OE33, OE33CisP and OE33CisR cells were cultured in RPMI 1640 supplemented with $2 \mathrm{mM}$ L-glutamine, $10 \%$ foetal bovine serum and $1 \%$ penicillin-streptomycin (Gibco) at $37^{\circ} \mathrm{C}$ in a humidified atmosphere of $5 \% \mathrm{CO}_{2}$. Cells were routinely mycoplasma tested. Isogenic OE33CisP and OE33CisR cell lines were age and passage matched.

\section{Surface and intracellular staining for flow cytometric analysis}


OE33CisP and OE33CisR cells were stained for flow cytometry using PVRL-1-AlexaFlour488, PVRL-4-PE, ULBP-3-APC, B7-H6-PE (R\&D, USA), MICA/B-PE-Vio770, HLA-E-FITC, 4-1BBL-PE-Vio770, PVR-APC (Miltenyi Biotec) and TRAIL-R2-PE (Biolegend).

For co-culture, OE33CisP and OE33CisR cells were trypsinised at $80 \%$ confluency and seeded at a density of $2 \times 10^{5}$ cells $/ \mathrm{ml}$ in RPMI supplemented with $10 \%$ FBS and $1 \%$ penicillin-streptomycin (Gibco). Peripheral blood mononuclear cells (PBMC) were isolated from the blood of healthy donors by density gradient centrifugation and resuspended in RPMI supplemented with 10\% FBS and 1\% penicillin-streptomycin (Gibco). PBMC were co-cultured at a 1:1 ratio with OE33CisP or OE33CisR cells for 24 hours.

Following co-culture, PBMC were stained for flow cytometry using the following surface antibodies; CD56FITC-Viobright, NKG2A-APC (Miltenyi-Biotec) CD3-APC-Cy7, NKG2D-PE-Cy5, NKP46-PE-Cy7, NKp30BV421, CD69-BV510, TIGIT-PE-Cy5, PD-1-PE-Cy7, TRAIL-APC, FasL-BV421, LAG3-PE-Cy7, CTLA-4-PE-Cy5 (Biolegend), A2AR-PE (Bio-techne) and TIM-3-AF647 (BD Biosciences).

For intracellular staining, cells were stimulated with $10 \mathrm{ng} / \mathrm{ml} \mathrm{PMA}$ and $1 \mu \mathrm{g} / \mathrm{ml}$ lonomycin for a total of four hours. CD107a (Biolegend) was added at this time to detect degranulation. After one hour, 10 $\mathrm{g} / \mathrm{ml}$ of Brefeldin A (Sigma) was added for the remaining three hours. Cells were stained for surface markers with CD56-FITC-Viobright (Miltenyi-Biotec) and CD3-APC-Cy7 (Biolegend). Cells were stained for intracellular markers with TNF-a-APC, IL-10-BV421 and IFN-y-BV510 (Biolegend) using the FIX\&PERM Cell Fixation and Permeabilization Kit (Nordic MUBio).

For the quantification of NKP30 ${ }^{+} \mathrm{NK}$ cells in OAC patient blood, whole blood was collected at a time point of surgical resection and was stained with CD56-FITC-Viobright (Miltenyi Biotec), CD3-APC-Cy7 and NKp30-BV421 (Biolegend). Red blood cells were lysed using BD Lysing Solution (BD Biosciences) as per manufacturer's instructions. NK cells were quantified as $\mathrm{CD} 56^{+} \mathrm{CD} 3^{-}$cells within the lymphocyte gate.

All samples were acquired using the BD FACS CANTO II (BD Biosciences) flow cytometer and analysed using FlowJo v10 software (Tree Star). NK cells were defined as $\mathrm{CD}^{2} 6^{+} \mathrm{CD}^{-}$in the lymphocyte gate.

\section{Treatment of OE33 cells with clinically relevant combinations of chemotherapy}

OE33 cells were seeded at a density of 5,000 cells $/ 200 \mu l$ media and treated for 48 hours with the chemotherapeutics from the FLOT $(0.8249 \mu \mathrm{M}$ 5-fluorouracil, $2 \mu \mathrm{M}$ oxaliplatin, $0.001 \mu \mathrm{M}$ docetaxel), CROSS $(0.001 \mu \mathrm{M}$ paclitaxel, $1000 \mu \mathrm{M}$ carboplatin, \pm 2 Gy irradiation) or MAGIC $(1.493 \mu \mathrm{M}$ epirubicin, $1.5 \mu \mathrm{M}$ cisplatin, $0.8249 \mu \mathrm{M} 5$-flurouracil) (Sigma) regimens to simulate the effects of the most commonly used chemotherapeutic regimens for OAC patients. $\mathrm{An} \mathrm{IC}_{25}$ of each treatment was used which resulted in $50 \%$ of OE33 cell viability, as determined by a CCK8 assay. Following treatment supernatants were collected.

\section{Quantification of soluble NKR Ligands in the OAC microenvironments and OAC patient serum}


OE33CisP and OE33CisR cells were pelleted and supernatants were collected and cryopreserved at $-80^{\circ} \mathrm{C}$. Supernatants from parent OE33 cells treated with clinically relevant regimes of chemoradiotherapy (CRT) as described above were collected and cryopreserved at $-80^{\circ} \mathrm{C}$. Human B7-H6 duo-set ELISA, human ULBP-3 duo-set ELISA (R\&D) and human MICA ELISA (ELISA Genie) were carried out as per manufacturer's instructions. For normalisation, total protein was determined from all cell lines using the Pierce $^{\text {TM }}$ BCA Protein Assay Kit (ThermoFisher) as per manufacturer's instructions. Absorbance values were measured at $595 \mathrm{~nm}$ using a VersaMax plate reader and SoftmaxPro 6.1 software from which a standard curve was generated to deduce total protein concentrations.

\section{Quantitative Real-time PCR}

Total RNA was isolated from cell lines using TRI Reagent (Molecular Research Center, USA). RNA yield and purity were determined using a Nanodrop-1000 spectrophotometer. Gene expression analysis of the NK cell ligands PVRL-1, PVRL-4, HLA-E, B7-H6 and ULBP3, was carried out in duplicate using the Luna Universal One-Step RT-qPCR Kit (New England BioLabs Inc), in duplicate, on the ABI 7500 qPCR system. B-actin was used to normalise for endogenous gene expression. Complementary DNA (CDNA) was generated by reverse transcription for $10 \mathrm{~min}$ at $55^{\circ} \mathrm{C}$. Real-time PCR was carried out by denaturation at $95^{\circ} \mathrm{C}$ for 1 min followed by 45 cycles consisting of denaturation at $95^{\circ} \mathrm{C}$ for 10 secs, primer extension at $60^{\circ} \mathrm{C}$ for 60 secs and a final elongation step at $60^{\circ} \mathrm{C}$ for $1 \mathrm{~min}$. Mean CT values were determined and differences in gene expression levels were calculated as fold-changes relative to controls. Primers were designed using the University of California Santa Cruz (UCSC) Genome Browser and synthesised commercially (Sigma-Aldrich). Primers sequences used are as follows: PVRL-1 FWD 5'TCTCGGCTTGACCGCATTCT-3', REV 5'-TGGTGGACTTCTGCCATGTGAC-3'; PVRL-4 FWD 5'GGAAGACTCTGGGAAGCAGG-3', REV 5'-GATGGAGTTCTCCCTGGTCAG-3'; HLA-E FWD 5'AAGACACATGCGTGGAGTGG-3'; REV 5'-TGTGATCTCCGCAGGGTAGA-3'; B7H6 FWD 5'TCCATTCATTGGTGGCCTAT-3', REV 5'-TGCAAAAGAATATGAGGTGCTCT-3'; ULBP3 FWD 5'GGCGGATGAAAGAGAAGTGG-3', REV 5'-TTGGGTTGAGCTAAGCCTGG-3', $\beta$-actin FWD 5'- TGTTTGAGACCTTCAACACCC-3', REV 5'- AGCACTGTGTTGGCGTACAG-3'.

\section{Patient Demographics}

Blood sera and whole blood were collected from a total of 30 consenting OAC patients attending the National Oesophageal and Gastric Centre at St. James's Hospital, Dublin [Table I]. The patient group included 22 males and 8 females, reflecting the predominance of OAC in males. The group ranged in age 48-83 years and had a mean age of 65.2 years. Within this cohort, $80 \%$ had received neoadjuvant CRT regimens. Tumour regression grade (TRG) was used to categorise patients as good responders (TRG 1-2) or poor responders (TRG 3-5) to chemo-radiotherapy [15]. The computed tomography defined visceral fat area (VFA) was calculated as described previously and categorised as obese when VFA was greater than $160 \mathrm{~cm}^{2}$ for men and $80 \mathrm{~cm}^{2}$ for women [16]. The mean VFA was $145.38 \mathrm{~cm}^{2}$, with $55 \%$ of patients measuring as viscerally obese. 


\section{Statistical Analysis}

Statistical analysis was performed using GraphPad Prism version 9 (GraphPad software). The statistical significance between groups was determined by a t test or one-way ANOVA with post-hoc Dunnett's or Tukey's test as appropriate. $P<0.05$ was considered significant.

\section{Results}

\section{NKR ligands are expressed at significantly lower levels by OE33CisR cells compared to OE33CisP cells}

The expression of NKR ligands on treatment-resistant OAC tumour cells was quantified to assess the susceptibility of treatment-resistant OAC tumour cells to NK cells. Here we report significantly lower frequencies of $\mathrm{B} 7-\mathrm{H6}^{+}$(OE33CisP vs. OE33CisR; 7.544\% vs. 2.955\%; $\left.\mathrm{p}=0.049\right)$, MICA/B ${ }^{+}$(OE33CisP vs. OE33CisR; $16.53 \%$ vs. $11.43 \% ; p=0.0008$ ) and ULBP-3+ (OE33CisP vs. OE33CisR; $10.78 \%$ vs. $4.150 \%$; $p=0.0080)$ cells within the OE33CisR population compared to the OE33CisP cell population (all $n=3)(F i g$. 1). Additionally, significantly lower frequencies of cells expressing the activation/inhibitory ligands $P V R L-$ 4 (OE33CisP vs. OE33CisR; $62.03 \%$ vs. 51.60\%; $p=0.0013$ ) and PVRL-1 (OE33CisP vs. OE33CisR; 17.33\% vs. $9.233 \%$; $p=0.0252$ ) (Fig. 1) were observed on the surface of OE33CisR cells, compared to OE33CisP cells.

In contrast, there were significantly lower proportions of OE33CisP cells expressing 4-1BBL (OE33CisP vs. OE33CisR; $11.28 \%$ vs. $15.07 \%$; $p=0.0144$ ) (Fig. 1) compared to OE33CisR cells. There were no significant differences in the frequencies of OE33CisP and OE33CisR cells expressing TRAIL-R2, HLA-E or PVR (Fig. 1).

\section{There are no significant differences in mRNA expression of NKR ligands between OE33CisR and OE33CisP cells}

To ascertain whether significantly lower surface expression of activating NKR ligands on OE33CisR cells was due, at least in part, to a downregulation of the genes encoding these ligands, mRNA levels were measured by RT-PCR. There were no significant differences in the expression levels of B7-H6, HLA-E, PVRL-1, PVRL-4 or ULBP-3 between OE33CisP and OE33CisR cells (Fig. 2). There was a trend however towards higher levels of PVRL-4 and ULBP-3 mRNA expression in OE33CisR cells relative to their parent counterparts.

\section{Standard-of-care chemotherapeutic regimens for OAC can modulate B7-H6 shedding by OE33 cells.}

To ascertain whether significantly lower surface expression of activating NKR ligands on OE33CisR cells was due to increased shedding, a panel of soluble NKR ligands were measured in the supernatants of the OE33CisP and OE33CisR cells (B7-H6, MICA/B and ULBP3). Similar B7-H6 concentration were observed in the supernatant of OE33CisP and OE33CisR cells, with both cell lines shedding less than 0.5pg B7-H6 per $\mu \mathrm{g} / \mathrm{ml}$ of protein (Fig. 3A). Furthermore, there were undetectable levels of MICA and ULBP-3 soluble 
protein in the cell line supernatant from OE33CisP and OE33CisR cell lines [data not shown]. These data suggest that the significantly lower surface expression of B7-H6, MICA/B and ULBP-3 by OE33CisR cell lines is not due to increased shedding of these ligands.

To examine B7-H6 ligand shedding in vivo, soluble B7-H6 levels were measured in the serum of OAC patients. Interestingly, there was a significant positive correlation between visceral fat area (VFA) and serum B7-H6 protein ( $r=0.8113, p=0.0044, n=10)$ (Fig. 3B). Interestingly, there were considerably lower frequencies of ${\mathrm{NKp} 30^{+}}^{+} \mathrm{NK}$ cells in the circulation of obese $(n=10)$ OAC patients, compared to their nonobese $(n=5)$ counterparts (Non-obese vs obese; $88.84 \%$ vs. $64.02 \%, p=0.06)$ (Fig. 3F). Moreover, higher levels of serum B7-H6 were detected in OAC patients with a higher tumour regression grade TRG (TRG 3$5, n=6)$ which is indicative of poor treatment response, compared to patients with a lower TRG (TRG 1-2, $\mathrm{n}=4$ ) (Low TRG vs. High TRG; $257.0 \mathrm{pg} / \mathrm{ml}$ vs. $967.2 \mathrm{pg} / \mathrm{ml}, \mathrm{p}=0.1021$ ) (Fig. 3D). There were significantly lower frequencies of NKp30 ${ }^{+}$NK cells in the circulation of OAC patients who had a higher TRG (TRG 3-5, $\mathrm{n}=4$ ) compared to those with a lower TRG (TRG 1-2, n=4) (Low TRG vs. High TRG; 79.63\% vs. 51.55\%, $\mathrm{p}=0.0259)(\mathbf{F i g} .3 \mathrm{H})$. These data suggest that OAC patients with greater VFA and poorer treatment response have higher levels of circulating $\mathrm{B} 7-\mathrm{H} 6$ and lower frequencies of circulating NKP30 ${ }^{+} \mathrm{NK}$ cells.

To determine whether treatment with neoadjuvant chemotherapy or CRT is associated with altered circulating levels of soluble B7-H6, serum B7-H6 levels were measured in OAC patients at time-points preand post-treatment. While there were substantially higher levels of soluble B7-H6 levels in the serum of patients post-FLOT, these were not significantly different (5-fluorouracil, oxaliplatin, docetaxel) (Pre-FLOT vs. Post-FLOT; $558 \mathrm{pg} / \mathrm{ml}$ vs. $733.8 \mathrm{pg} / \mathrm{ml}, \mathrm{n}=5$ ). Furthermore, there were no significant differences observed in the soluble B7-H6 levels in the serum of patients prior to CROSS (Pre-CROSS), compared to the Post-CROSS time-point (paclitaxel, carboplatin, \pm 2 Gy irradiation) (Pre-CROSS vs. Post-CROSS; $561.1 \mathrm{pg} / \mathrm{ml}$ vs. $535.68 \mathrm{pg} / \mathrm{ml}, \mathrm{n}=4$ ) (Fig. 3C).

Interestingly, there were significantly lower frequencies of $\mathrm{NKp}^{+} \mathrm{NK}^{+}$cells in the circulation of OAC patients who received FLOT $(n=9)$, compared to those who received CROSS $(n=4)$ (FLOT vs. CROSS; $48.99 \%$ vs. $86.55 \%, p=0.0121)$ or were treatment naïve $(n=4)$ at the time of surgical resection (FLOT vs. Naïve; $48.99 \%$ vs. $82.4 \%, p=0.0247$ ) (Fig. $3 H$ ). Similarly, there were significantly less NKG2D ${ }^{+}$NK cells in the circulation of OAC patients who received FLOT $(n=10)$, compared to those who received CROSS $(n=4)$ (FLOT vs. CROSS; $62.52 \%$ vs. $87.60 \%, p=0.05)$ or were treatment naïve $(n=4)$ at the time of surgical resection (FLOT vs. Naïve; $62.52 \%$ vs. $90.03 \%$, p= 0.0335) (Fig. 3l). Furthermore, there were significantly less NKp46 ${ }^{+}$NK cells in the circulation of OAC patients who received FLOT $(n=10)$, compared to those who received CROSS ( $n=4$ ) (FLOT vs. CROSS; $62.8 \%$ vs. $96.15 \%, p=0.0046)$. There appears to be considerably less NKp46 ${ }^{+} \mathrm{NK}$ cells in the circulation of OAC patients who were treatment naïve $(n=4)$ at the time of surgical resection compared to those who received FLOT (FLOT vs. Naïve; $62.8 \%$ vs. $82.85 \%$, $\mathrm{p}=0.08$ ) (Fig. 3J).

To ascertain whether treatment with clinically relevant chemotherapeutic combinations altered B7-H6 shedding into the treatment-naïve OAC tumour microenvironment, OE33 cells were treated with FLOT (5- 
fluorouracil, oxaliplatin, docetaxel), CROSS (paclitaxel, carboplatin, \pm 2 Gy irradiation), and MAGIC (epirubicin, cisplatin, 5-fluorouracil) chemo-radiotherapy regimens. Interestingly, there was a significant increase in soluble B7-H6 in the supernatant of OE33 cells following treatment with FLOT chemotherapy regimen, compared to vehicle control (Vehicle control vs. FLOT chemotherapy; $483.6 \mathrm{pg} / \mathrm{ml}$ vs. $784 \mathrm{pg} / \mathrm{ml}$; $\mathrm{p}=0.0034$ ) (Fig. 3E). In contrast, significant decreases in soluble B7-H6 were observed following treatment with CROSS chemotherapy (Vehicle control vs. CROSS chemotherapy; $483.6 \mathrm{pg} / \mathrm{ml}$ vs. $177.8 \mathrm{pg} / \mathrm{ml}$, $\mathrm{p}=0.0031$ ), and CROSS chemoradiotherapy (Vehicle control vs. CROSS chemoradiotherapy; $483.6 \mathrm{pg} / \mathrm{ml}$ vs. $221 \mathrm{pg} / \mathrm{ml} ; \mathrm{p}=0.0077$ ) (Fig. 3E).

\section{Significantly lower frequencies of NK cells express activating NKRs following co-culture with OE33CisP and OE33CisR cells}

To elucidate the effects of cisplatin-resistant OAC tumours on the phenotype of NK cells, proportions of cells expressing activating NKRs were assessed following co-culture with OE33CisP or OE33CisR cells. Our data revealed lower frequencies of ${\mathrm{NKp} 30^{+}}^{+} \mathrm{NK}$ cells following co-culture with OE33CisR cells compared to NK cells cultured alone ( $n=3$; Media only vs. OE33CisR; $96.87 \%$ vs. 92.87\%; $p=0.0181$ ) (Fig. 4A). Similarly, there were significantly lower frequencies of $\mathrm{NKp}^{+} 6^{+} \mathrm{NK}$ cells following co-culture with OE33CisR cells compared to NK cells cultured alone ( $n=5$; Media only vs. OE33CisR; $93.26 \%$ vs. $70.64 \%$; $\mathrm{p}=0.0020$ ) (Fig. 4B). It appears that co-culture with the cisplatin-sensitive OE33CisP cells also decreased the frequencies of NKp46 NK cells, compared to those left untreated ( $n=5$; Media only vs. OE33CisP; $93.26 \%$ vs. $72.56 \%$; $p=0.06$ ) (Fig. 4B). There were no significant differences in the frequencies of NKG2D ${ }^{+}$ NK cells following co-culture with OE33CisP or OE33CisR cells (Fig. 4C).

\section{Significantly higher frequencies of NK cells expressing TIGIT following co-culture with OE33CisR cells.}

The frequencies of NK cells expressing the immune checkpoint TIM-3 were significantly lower following co-culture with OE33CisP ( $n=3$, media only vs. OE33CisP; $28.9 \%$ vs. $11.03 \%, p=0.0334$ ) and CisR cells ( $n=3$, media only vs. OE33CisR; $28.9 \%$ vs. $12 \%, p=0.0428$ ) compared to those cultured alone (Fig 5A). Following co-culture of NK cells with OE33CisP cells, the frequencies of NK cells expressing the inhibitory receptor NKG2A was significantly decreased compared to NK cells cultured alone ( $n=5$; media only vs. OE33CisP; 79.78\% vs. 66.98\%, p=0.0082) (Fig. 5B). Interestingly, there were significantly higher frequencies of $\mathrm{TIGIT}^{+} \mathrm{NK}$ cells following co-culture with the cisplatin-resistant OE33CisR cells, compared to NK cells cultured alone ( $n=5$, media only vs. OE33CisR; $73.06 \%$ vs. $82.20 \% ; p=0.0421$ ) (Fig. 5C). No changes were observed in the frequencies of $\mathrm{A} 2 \mathrm{AR}{ }^{+}, \mathrm{PD}-1^{+}, \mathrm{CTLA}-4^{+}, \mathrm{LAG}-3^{+}$or $\mathrm{CD} 69^{+} \mathrm{NK}$ cells following co-culture with OE33CisP or OE33CisR cells (Fig. 5D-H).

\section{Significantly higher frequencies of NK cells expressing the death receptor ligands TRAIL and FasL following culture with cisplatin-resistant OAC cells.}

There were significantly higher frequencies of $\mathrm{TRAIL}^{+} \mathrm{NK}$ cells following co-culture with OE33CisR cells, compared to NK cells cultured alone ( $n=3$; media only vs. OE33CisR; $49.50 \%$ vs. $64.23 \%$; $p=0.0019$ ) (Fig. 
6A). Furthermore, there were significantly higher frequencies of NK cells expressing the death receptor ligand FasL following culture with both OE33CisP ( $n=3$; media only vs. OE33CisP; $2.203 \%$ vs. $91.43 \%$; $\mathrm{p}=0.0065$ ) and OE33CisR cells (media only vs. OE33CisR; $2.203 \%$ vs. $94.83 \%$; $p=0.0005$ ) (Fig. 6B). While not significant, the ability of NK cells to degranulate, as indicated by CD107a expression, may be increased following co-culture with OE33CisP cells ( $n=3-5$; media alone vs. OE33CisP; $14.79 \%$ vs. $49.40 \%$; $\mathrm{p}=0.07)$ (Fig. 6C).

\section{NK cell cytokine production is significantly altered following co-culture with OAC cells.}

To elucidate the functional effects of co-culture with OE33CisP and OE33CisR cells on NK cells, NK cell production of the pro-inflammatory cytokines IFN- $\gamma$ and TNF-a, and the anti-inflammatory cytokine IL-10 was examined. Interestingly, while there are significantly more IFN- $\gamma^{+} \mathrm{NK}$ cells following co-culture with OE33CisP ( $n=3$, media alone vs. OE33CisP; $24.67 \%$ vs. $85.73 \%$; $p=0.0100)$ and OE33CisR cells $(n=6$, media alone vs. OE33CisR; $24.67 \%$ vs. $66.25 \%$; $p=0.0336$ ) compared to those cultured alone, the increases in IFN- ${ }^{+} \mathrm{NK}$ cells are considerably lower when cultured with the resistant cells, compared to the sensitive cells (Fig. 7A). The frequencies of $\mathrm{IL}-10^{+} \mathrm{NK}$ cells were also increased following co-culture with OE33CisP cells, compared to those cultured alone ( $n=3$, media alone vs. OE33CisP; $61.93 \%$ vs. $90.97 \%$; $\mathrm{p}=0.05$ ) (Fig. 7B). There were no changes in the frequencies of TNF-a producing NK cells following coculture with OE33CisP or OE33CisR cells (Fig. 7C).

\section{Discussion}

Previous work by our group has uncovered altered NK cell frequency and function in OAC patients and we propose that NK cell therapy might be utilised to restore NK cell-mediated anti-tumour immunity for this cohort, who face dismal survival rates and a paucity of treatment options $[17,18]$. However, with advances in NK cell-based immunotherapies, it is prudent to be aware that many OAC patients will have previous exposure to standard-of-care treatments and an overwhelming majority of OAC patients may have treatment-resistant tumours. As such, it is imperative that we characterise these tumours to assess their susceptibility and suitability for NK cell-based therapies.

To gauge susceptibility to NK cell-mediated responses and indeed to NK cell therapy, we characterised the NKR ligand expression profile of our in-house cisplatin-resistant OAC cell line model OE33CisR and its cisplatin-sensitive counterpart, OE33CisP. Here, we identified significantly lower surface expression of NKR activating ligands B7-H6, MICA/B and ULBP-3 and activating/inhibitory NKR ligands PVRL-1 and PVRL-4 on OE33CisR cells compared to cisplatin-sensitive OE33CisP cells. Such diminished NKR ligand expression suggests that cisplatin-resistant OAC tumours may be less susceptible to NK cell-mediated killing than their cisplatin-sensitive counterparts.

Downregulation in gene expression of the NKG2D ligands, ULBP-1, ULBP-3 and MICA/B has been shown in cisplatin-resistant lung cancer cell lines compared to their parental counterparts, with mitogenactivated protein kinase kinase/extracellular signal-regulated kinase (MAPK/Erk) signalling implicated in 
this reduced ligand expression [19]. We hypothesised that the differences observed in NKR ligand surface expression between OE33CisP and OE33CisR may be due to alterations at the gene expression level. Our data however showed no significant differences in the levels of B7-H6, HLA-E, PVRL-1, PVRL-4 and ULBP3 mRNA expression between OE33CisP and OE33CisR cells.

While there has been success in utilising cellular based immunotherapies in the treatment of haematological malignancies, solid tumours pose additional challenges [20]. The solid tumour microenvironment (TME) is one such challenge and identifying the factors within the TME that may contribute to the reduced efficacy of cellular therapies is imperative to ensuring their success. Cleavage of NK activating ligands B7-H6 and MICA/B expressed on tumour cells and the subsequent release of these soluble ligands is a strategy through which tumour cells evade NK cell-mediated immune surveillance and thus may dampen the effects of NK cell therapies [21-23]. Since NKR ligands were not altered at a transcriptional level, we proposed that ligands were expressed but subsequently shed into the TME of treatment-resistant tumour cells. Our in vitro data did not support this hypothesis and the lower surface expression of B7-H6, MICA/B and ULBP-3 by OE33CisR cells was not paralleled by significantly higher soluble NKR ligand levels in the OE33CisR cell supernatant.

B7-H6 is an activating ligand for the receptor NKp30 and has gained much traction as a potential prognostic marker in multiple malignancies [24]. It is expressed preferentially on tumour cells, thus centring it as an important target for NK cell based therapies [24]. Interaction of NKp30 with its ligand B7$\mathrm{H} 6$ can modulate the downregulation of NKp30 on the surface of NK cells [25]. Soluble B7-H6 has been shown to contribute to defective expression of NKp30 on circulating NK cells from hepatocellular carcinoma, ovarian carcinoma and neuroblastoma patients, further linking elevated B7-H6 with NK cell dysfunction [26, 27, 25]. Indeed, soluble B7-H6 has been proposed as a biomarker for several cancers. In the case of gastrointestinal stromal tumours, detectable levels of serum B7-H6 were predictive of a poor treatment response and worse outcome for patients [28]. In neuroblastoma, B7-H6 levels inversely correlate with NKp30 surface expression suggesting that B7-H6 can act in the periphery by engaging the NKp30 receptor and thus reducing NKp30-dependent NK cell functions [29]. B7-H6 expression correlated with unfavourable prognosis for T-lymphoblastic lymphoma patients [30]. In patients with acute myeloid leukaemia an NKp30 high phenotype was predictive of a better outcome for patients [31].

Interestingly, our clinical analysis of patient serum samples from treatment responders and nonresponders revealed the highest levels of soluble B7-H6 in the serum of OAC patients with a higher tumour regression grade TRG (TRG 3-5) following CRT, which is indicative of poor treatment response. These trends would suggest that poor treatment responders have higher circulating levels of soluble B7$\mathrm{H6}$, which might be indicative of higher intratumoural levels and a possible immune evasion strategy within treatment-resistant OAC tumours. The higher levels of circulating soluble B7-H6 in poor treatment responders were paralleled by significantly lower frequencies of circulating NK cells expressing its cognate receptor, NKp30. NKp30 ${ }^{\text {low }}$ NK cells have been shown to exhibit impaired cytolytic function and IFN- $\gamma$ production thus suggesting that OAC patients who are poor treatment responders have diminished NK cell activity, possibly mediated by enhanced levels of soluble B7-H6 [26]. 
There is compelling evidence to suggest chemotherapeutic agents modulate cellular stress pathways, and could impact NK cell expression of activating and inhibitory ligands [32]. Determining the chemotherapeutic effects on NKR ligand shedding by tumour cells can inform the timing of NK cell therapies with first-line treatments in OAC. To ascertain whether chemotherapeutic combination regimens used to treat OAC could alter NKR ligand shedding in the TME, treatment-naïve OAC tumour cells were treated with FLOT, CROSS and MAGIC [33]. Our results demonstrate that CROSS can reduce B7-H6 shedding in OE33 cells. This places CROSS as a preferable regimen to potentially combine with NK cell therapies to minimise immune evasion strategies mediated through B7-H6 shedding. In contrast, the FLOT regimen induced $\mathrm{B} 7-\mathrm{H} 6$ shedding by $\mathrm{OE} 33$ cells, hence this regimen may hinder NK cell responses and indeed NK cell therapy. Indeed, here we report significantly lower frequencies of NKp30 $0^{+} \mathrm{NK}$ cells in the circulation of OAC patients who received the FLOT treatment regimen compared to those who received the CROSS regimen or were treatment naïve at surgical resection. This is paralleled by significantly lower frequencies of NKG2D $\mathrm{D}^{+}$and $\mathrm{NKp} 46^{+} \mathrm{NK}$ cells in OAC patient blood following treatment with FLOT compared to CROSS or no treatment. Exposure to soluble B7-H6 has been shown to cause a decrease in expression of these activating receptors on NK cells, indicating B7-H6 has wide reaching effects beyond its own cognate receptor [34]. Interestingly, the chemotherapeutic 5-FU which is a component of the FLOT regimen has been shown to increase B7-H6 expression on tumour cells [35]. Furthermore, 5-FU is also a known activator of ADAM17, the sheddase which causes the shedding of B7$\mathrm{H} 6$ from the tumour cell surface [36]. This suggests that FLOT chemotherapy regimen may both increase the expression of $\mathrm{B} 7-\mathrm{H} 6$ on the tumour cell surface and aid in mediating the shedding of $\mathrm{B} 7-\mathrm{H} 6$ into the tumour microenvironment which may lead to defective NK cell function.

Interestingly, serum levels of B7-H6 were not significantly altered following first line treatments in OAC patients and circulating B7-H6 levels were not significantly higher following FLOT. Since such post-CRT serum samples were collected on the day of surgery and 6 weeks after last dose of chemotherapy, these observations suggest that either the stimulatory or inhibitory effects of chemotherapy on B7-H6 shedding are not maintained after treatment has ceased, or that the serum may not be a useful indicator of chemotherapy-induced NKR ligand shedding within the TME of OAC patients. Overall our data suggest that analysis of NKp30 expression on circulating NK cells may be superior to serum B7-H6 as a blood biomarker for treatment response and may allow for the identification of OAC patients who are treatment-resistant. Furthermore, NKP30 may be used to inform the use of chemoradiotherapy regimens which do not promote an inhibitory NK cell phenotype and thus would be more appropriate to pair with an NK cell-based immunotherapy.

Interestingly, here we report a significant correlation between increasing visceral adiposity and B7-H6 serum levels in OAC patients. Furthermore, frequencies of NK cells expressing the activating receptor NKp30 were significantly lower in obese OAC patients, compared to their non-obese counterparts, as stratified by VFA. OAC has a strong association with obesity, and we have reported extensive alterations in both the phenotype and function of NK cells in these obesity-associated cancer patients, with the most obese patients having the lowest number of intratumoural NK cells $[17,37,18]$. This present study 
suggests that NKR ligand shedding is exacerbated in viscerally obese OAC patients and may contribute to diminished NKp30 expression on circulating NK cells and compromised anti-tumour responses in these patients. These data provide further evidence that visceral obesity is an important pathological entity with wide-reaching effects [38].

Despite no obvious differences in the shedding of several key NKR ligands in our cell line models of cisplatin-resistant OAC tumours, our data revealed that co-culture with OE33CisP and OE33CisR cells could significantly modulate surface expression of NKRs on primary human NK cells. The frequencies of $\mathrm{NKp} 46^{+}$and $\mathrm{NKp} 30^{+} \mathrm{NK}$ cells were significantly lower following co-culture with OE33CisR cells. Soluble factors such as TGF- $\beta$, activin-A and adenosine are known to inhibit NK cell activity and mediate tumour evasion and thus may be contributing to these alterations in NK cell phenotype following co-culture with the cisplatin-resistant OAC cells [39]. These results demonstrate that treatment-resistant OAC tumour cells can decrease the frequencies of NK cells expressing activating receptors, and thus may hinder recognition of tumour cells and NK cell-mediated killing. A shift in the phenotype of NK cells via alterations in NKR expression has previously been reported to dampen immune surveillance and cytotoxic abilities of NK cells, hence facilitating tumour growth in ovarian cancer [40]. While the mediators of such reductions were not identified in this study, our group have previously reported that OE33CisR cells have altered inflammatory chemokines and cytokines profiles [14]. In particular, the production of key NK cell cytokines IL-2, IL-10 and TNF- $a$ is significantly lower from OE33CisR cells compared to their OE33CisP counterparts [14].

Our data also revealed that frequencies of immune checkpoint $\mathrm{TIGIT}^{+}$NK cells were increased following culture with OE33CisR cells. Interestingly, the frequencies of NK cells expressing the inhibitory receptor NKG2A were significantly decreased following co-culture with OE33CisP cells, whilst their frequencies were maintained following co-culture with the cisplatin-resistant OE33CisR cells. High expression of surface TIGIT contributes to NK cell exhaustion in solid tumours and therefore blockade can enhance NK cell-mediated responses [41, 42]. Combination of TIGIT blockade with the anti-human epidermal growth factor receptor 2 antibody trastuzumab has been shown to enhance NK cell-mediated antibodydependent cellular cytotoxicity [43]. These data provide new evidence that targeting the immune checkpoints TIGIT may be particularly effective in the setting of treatment-resistant OAC tumours. The frequencies of TIM- $3^{+} \mathrm{NK}$ cells were significantly decreased following culture with both OE33CisP cells and OE33CisR cells. TIM-3 is a known NK cell maturation marker which possesses both activating and inhibitory functions and has been shown to both impair NK cell mediated cytotoxicity and enhance IFN- $\gamma$ production $[44,45]$. In line with results reported here, exposure to glioblastoma and prostate cancer cells resulted in a downregulation of TIM-3 [46]. Interestingly, while previous studies have reported a correlation between TIM-3 expression and decreased cytotoxicity, we report significantly higher frequencies of death receptor ligand and IFN-y expressing NK cells following co-culture with both cell lines, suggesting TIM-3 mediated effects on these markers are not immediate [46]. Furthermore, it suggests in this setting that TIM-3 may have an inhibitory role for NK cells in OAC and that cancer cell mediated decreases in the expression of this marker may facilitate enhanced NK cell function in both the cisplatin-sensitive and 
resistant OAC tumour. The multi-faceted role of TIM-3 in relation to NK cell phenotype and function in OAC warrants further exploration.

Despite decreases in activating NKR expression following co-culture with OE33CisR cells, our data revealed that frequencies of death receptor ligands $\mathrm{TRAIL}^{+}$and $\mathrm{FasL}^{+} \mathrm{NK}$ cells were significantly increased. These data suggest that the OAC TME promotes the upregulation of key death receptor ligands on the surface of NK cells and provides a glimpse into the potential mechanisms NK cells employ to mediate elicit their effects against such tumours. Furthermore, our findings uncovered significantly higher frequencies of pro-inflammatory IFN- $\gamma^{+} \mathrm{NK}$ cells following culture with both OE33CisP and OE33CisR cells, further suggesting that the cisplatin-resistant OAC tumour does not significantly impede NK cell function. Indeed, NK cell-derived IFN-y plays a pivotal role in tumour cell cytolysis via promotion of conjugate formation [47] and our group has previously reported that the OAC tumour is enriched in this pro-inflammatory cytokine, which has been shown to mediate death receptor ligand expression [48, 49]. Additionally, it appears that neither cisplatin-sensitive nor cisplatin-resistant OAC tumour cells hamper NK cell degranulation, as indicated by CD107a expression and may even increase it. Overall, these data suggest that the introduction of NK cells to the OAC tumour microenvironment does not irrevocably alter NK cell function and that such tumours may be amenable to NK cell therapies.

\section{Conclusion}

In this study, we report for the first time, the profiling of NKR ligand expression in cisplatin-sensitive and cisplatin-resistant OAC cells, identifying unique ligand expression profiles in this cancer type. While coculture with treatment-resistant OAC tumour cells alters the frequencies of NK cells expressing activating and inhibitory NKRs, immune checkpoints and death receptors, they do not negatively modulate IFN- $Y$ production or degranulation of these potent anti-tumour cells. Such altered NKR profiles are mirrored in OAC patients in which poor treatment responders have significantly lower numbers of key NKP30${ }^{+} \mathrm{NK}$ cell subset. Interestingly, we have identified that the FLOT chemotherapy regimen induces NKP30 ligand B7$\mathrm{H} 6$ shedding by OAC tumour cells and thus their subsequent susceptibility to NK cells and NK cell-based therapies. In line with this, the frequencies of NK cells expressing NKp30 are significantly diminished following FLOT chemotherapy in OAC patients. Finally our data identify that B7-H6 serum levels correlate with visceral adiposity suggesting that obesity exacerbates such ligand shedding and the resulting NK cell modulation. As such, it is integral that the impact of both first-line treatments and the obese environment are considered for the successful design of NK cell-based therapies.

\section{Declarations}

\section{Funding}

This work was funded by Enterprise Ireland Award (CS20192036), Breakthrough Cancer Research (BCR2019-02-PhD-TCD) and the M.Sc. Translational Oncology, Trinity Translational Medicine Institute, Trinity College Dublin. 


\section{Conflicts of interest/Competing interests}

The authors have no conflicts of interest to declare.

\section{Ethics approval}

The work was performed in accordance with The Code of Ethics of the World Medical Association (Declaration of Helsinki) for experiments involving humans. Patients provided informed consent for sample and data acquisition and the study received full ethical approval from the St. James's Hospital Ethics Review Board. Patient samples were pseudonymized to protect the privacy rights of the patients.

\section{Consent to participate}

All patients provided informed consent for sample and data acquisition.

\section{Consent for publication}

All patients provided explicit and informed consent for their coded data and results to be reported in scientific journals.

\section{Availability of data and material}

Data sharing not applicable to this article as no datasets were generated or analysed during the current study.

\section{Code availability}

Not applicable

\section{Authors' contributions}

E. Mylod and E. McKenna performed the experiments and data analysis, carried out statistical analysis and co-wrote the manuscript. M. Davern assisted with experiments and generated chemotherapy-treated supernatants. M.P. Barr conducted gene expression experiments. S.G. Maher and B.A.S. Bibby generated the OE33CisP and OE33CisR cell lines. N.E. Donlon and J.V. Reynolds consented patients for procurement of samples. N.E. Donlon measured anthropometric variables. A. Bhardwaj retrieved clinical data and samples. J. Lysaght assisted in writing and revision of the manuscript. M.J. Conroy designed and directed the project, supervised the conduction of experiments and analysis, and co-wrote the manuscript.

\section{Précis}

We report that chemotherapy-resistant OAC tumour cells do not significantly hamper NK cell function and might be amenable to NK cell therapy. We also report that FLOT chemotherapy induces B7-H6 shedding by tumour cells and may promote immune evasion. 


\section{References}

1. Society AC. Survival Rates for Esophageal Cancer. https://www.cancer.org/cancer/esophaguscancer/detection-diagnosis-staging/survival-rates.html.

2. !!! INVALID CITATION !!! [2, 3].

3. Moehler M, Shitara K, Garrido M, Salman P, Shen L, Wyrwicz L, et al. LBA6_PR Nivolumab (nivo) plus chemotherapy (chemo) versus chemo as first-line (1L) treatment for advanced gastric cancer/gastroesophageal junction cancer (GC/GEJC)/esophageal adenocarcinoma (EAC): First results of the CheckMate 649 study. Annals of Oncology. 2020.

4. Muro K, Chung HC, Shankaran V, Geva R, Catenacci D, Gupta S, et al. Pembrolizumab for patients with PD-L1-positive advanced gastric cancer (KEYNOTE-012): a multicentre, open-label, phase 1b trial. Lancet Oncol. 2016 Jun;17(6):717-26.

5. Habif G, Crinier A, André P, Vivier E, Narni-Mancinelli E. Targeting natural killer cells in solid tumors. Cellular \& Molecular Immunology. 2019 2019/05/01;16(5):415-22.

6. Trapani JA, Smyth MJ. Functional significance of the perforin/granzyme cell death pathway. Nature Reviews Immunology. 2002 10/01/online;2:735.

7. Vitale M, Della Chiesa M, Carlomagno S, Romagnani C, Thiel A, Moretta L, et al. The small subset of CD56brightCD16- natural killer cells is selectively responsible for both cell proliferation and interferon-gamma production upon interaction with dendritic cells. Eur J Immunol. 2004 Jun;34(6):1715-22.

8. Mandal A, Viswanathan C. Natural killer cells: In health and disease. Hematology/Oncology and Stem Cell Therapy. 2015 2015/06/01/;8(2):47-55.

9. Vivier E, Nunes JA, Vely F. Natural killer cell signaling pathways. Science. 2004 Nov 26;306(5701):1517-9.

10. Waldman AD, Fritz JM, Lenardo MJ. A guide to cancer immunotherapy: from T cell basic science to clinical practice. Nature Reviews Immunology. 2020 2020/11/01;20(11):651-68.

11. Natural killer cells for cancer immunotherapy: a new CAR is catching up. EBioMedicine. 2019;39:1-2.

12. Souza-Fonseca-Guimaraes F, Cursons J, Huntington ND. The Emergence of Natural Killer Cells as a Major Target in Cancer Immunotherapy. Trends Immunol. 2019 Feb;40(2):142-58.

13. Lynam-Lennon N, Heavey S, Sommerville G, Bibby BAS, Ffrench B, Quinn J, et al. MicroRNA-17 is downregulated in esophageal adenocarcinoma cancer stem-like cells and promotes a radioresistant phenotype. Oncotarget. 2017;8.

14. Buckley AM, Bibby BA, Dunne MR, Kennedy SA, Davern MB, Kennedy BN, et al. Characterisation of an Isogenic Model of Cisplatin Resistance in Oesophageal Adenocarcinoma Cells. Pharmaceuticals (Basel). 2019 Feb 20;12(1).

15. Guo K, Cai L, Zhang Y, Zhu J-F, Rong T-H, Lin P, et al. The predictive value of histological tumor regression grading (TRG) for therapeutic evaluation in locally advanced esophageal carcinoma treated with neoadjuvant chemotherapy. Chinese journal of cancer. 2012;31(8):399-408. 
16. Doyle SL, Bennett AM, Donohoe CL, Mongan AM, Howard JM, Lithander FE, et al. Establishing computed tomography-defined visceral fat area thresholds for use in obesity-related cancer research. Nutr Res. 2013 Mar;33(3):171-9.

17. Conroy MJ, Fitzgerald V, Doyle SL, Channon S, Useckaite Z, Gilmartin N, et al. The microenvironment of visceral adipose tissue and liver alter natural killer cell viability and function. J Leukoc Biol. 2016 Dec;100(6):1435-42.

18. Mylod E, Melo AM, Donlon NE, Davern M, Bhardwaj A, Reynolds JV, et al. Fractalkine Elicits Chemotactic, Phenotypic, and Functional Effects on CX3CR1(+)CD27(-) NK Cells in ObesityAssociated Cancer. J Immunol. 2021 Jul 28.

19. Yang L, Shen M, Xu LJ, Yang X, Tsai Y, Keng PC, et al. Enhancing NK cell-mediated cytotoxicity to cisplatin-resistant lung cancer cells via MEK/Erk signaling inhibition. Scientific reports. 2017;7(1):7958-58.

20. Dong S, Ghobrial IM. Immunotherapy for hematological malignancies. Journal of life sciences (Westlake Village, Calif). 2019;1(1):46-52.

21. Schlecker E, Fiegler N, Arnold A, Altevogt P, Rose-John S, Moldenhauer G, et al. Metalloproteasemediated tumor cell shedding of $\mathrm{B} 7-\mathrm{H} 6$, the ligand of the natural killer cell-activating receptor NKp30. Cancer Res. 2014 Jul 1;74(13):3429-40.

22. Pardoll DM. Distinct mechanisms of tumor resistance to NK killing: of mice and men. Immunity. 2015;42(4):605-06.

23. Xing S, Ferrari de Andrade L. NKG2D and MICA/B shedding: a 'tag game' between NK cells and malignant cells. Clin Transl Immunology. 2020;9(12):e1230.

24. Chen Y, Mo J, Jia X, He Y. The B7 Family Member B7-H6: a New Bane of Tumor. Pathol Oncol Res. 2018 Oct;24(4):717-21.

25. Mantovani S, Oliviero B, Lombardi A, Varchetta S, Mele D, Sangiovanni A, et al. Deficient Natural Killer Cell NKp30-Mediated Function and Altered NCR3 Splice Variants in Hepatocellular Carcinoma. Hepatology. 2019 Mar;69(3):1165-79.

26. Pesce S, Tabellini G, Cantoni C, Patrizi O, Coltrini D, Rampinelli F, et al. B7-H6-mediated downregulation of NKp30 in NK cells contributes to ovarian carcinoma immune escape. Oncoimmunology. 2015 Apr;4(4):e1001224.

27. Semeraro M, Rusakiewicz S, Zitvogel L, Kroemer G. Natural killer cell mediated immunosurveillance of pediatric neuroblastoma. Oncoimmunology. 2015 Nov;4(11):e1042202.

28. Rusakiewicz S, Perier A, Semeraro M, Pitt JM, Pogge von Strandmann E, Reiners KS, et al. NKp30 isoforms and NKp30 ligands are predictive biomarkers of response to imatinib mesylate in metastatic GIST patients. Oncoimmunology. 2017;6(1):e1137418.

29. Semeraro M, Rusakiewicz S, Minard-Colin V, Delahaye NF, Enot D, Vély F, et al. Clinical impact of the NKp30/B7-H6 axis in high-risk neuroblastoma patients. Sci Transl Med. 2015 Apr 15;7(283):283ra55.

30. Yuan L, Sun L, Yang S, Chen X, Wang J, Jing H, et al. B7-H6 is a new potential biomarker and therapeutic target of T-lymphoblastic lymphoma. Ann Transl Med. 2021 Feb;9(4):328. 
31. Chretien AS, Fauriat C, Orlanducci F, Rey J, Borg GB, Gautherot E, et al. NKp30 expression is a prognostic immune biomarker for stratification of patients with intermediate-risk acute myeloid leukemia. Oncotarget. $2017 \mathrm{Jul}$ 25;8(30):49548-63.

32. Zingoni A, Fionda C, Borrelli C, Cippitelli M, Santoni A, Soriani A. Natural Killer Cell Response to Chemotherapy-Stressed Cancer Cells: Role in Tumor Immunosurveillance. Front Immunol. 2017 2017-September-25;8(1194).

33. Donohoe CL, Reynolds JV. Neoadjuvant treatment of locally advanced esophageal and junctional cancer: the evidence-base, current key questions and clinical trials. J Thorac Dis. 2017;9(Suppl 8):S697-S704.

34. Ponath V, Hoffmann N, Bergmann L, Mäder C, Alashkar Alhamwe B, Preußer C, et al. Secreted Ligands of the NK Cell Receptor NKp30: B7-H6 Is in Contrast to BAG6 Only Marginally Released via Extracellular Vesicles. Int J Mol Sci. 2021 Feb 22;22(4).

35. Cao G, Wang J, Zheng X, Wei H, Tian Z, Sun R. Tumor Therapeutics Work as Stress Inducers to Enhance Tumor Sensitivity to Natural Killer (NK) Cell Cytolysis by Up-regulating NKp30 Ligand B7-H6. J Biol Chem. 2015 Dec 11;290(50):29964-73.

36. Kyula JN, Van Schaeybroeck S, Doherty J, Fenning CS, Longley DB, Johnston PG. Chemotherapyinduced activation of ADAM-17: a novel mechanism of drug resistance in colorectal cancer. Clin Cancer Res. 2010 Jul 1;16(13):3378-89.

37. Lauby-Secretan B, Scoccianti C, Loomis D, Grosse Y, Bianchini F, Straif K. Body Fatness and Cancer-Viewpoint of the IARC Working Group. N Engl J Med. 2016 Aug 25;375(8):794-8.

38. Deng T, Lyon CJ, Bergin S, Caligiuri MA, Hsueh WA. Obesity, Inflammation, and Cancer. Annu Rev Pathol. 2016 May 23;11:421-49.

39. Huntington ND, Cursons J, Rautela J. The cancer-natural killer cell immunity cycle. Nature Reviews Cancer. 2020 2020/06/24.

40. Carlsten M, Norell H, Bryceson YT, Poschke I, Schedvins K, Ljunggren HG, et al. Primary human tumor cells expressing CD155 impair tumor targeting by down-regulating DNAM-1 on NK cells. J Immunol. 2009 Oct 15;183(8):4921-30.

41. Sun $C$, Xu J, Huang Q, Huang M, Wen H, Zhang $C$, et al. High NKG2A expression contributes to NK cell exhaustion and predicts a poor prognosis of patients with liver cancer. Oncoimmunology. 2016;6(1):e1264562-e62.

42. Zhang Q, Bi J, Zheng X, Chen Y, Wang H, Wu W, et al. Blockade of the checkpoint receptor TIGIT prevents NK cell exhaustion and elicits potent anti-tumor immunity. Nature Immunology. 2018 2018/07/01;19(7):723-32.

43. Xu F, Sunderland A, Zhou Y, Schulick RD, Edil BH, Zhu Y. Blockade of CD112R and TIGIT signaling sensitizes human natural killer cell functions. Cancer immunology, immunotherapy : Cll. 2017;66(10):1367-75.

44. Gleason MK, Lenvik TR, McCullar V, Felices M, O'Brien MS, Cooley SA, et al. Tim-3 is an inducible human natural killer cell receptor that enhances interferon gamma production in response to 
galectin-9. Blood. 2012 Mar 29;119(13):3064-72.

45. Ndhlovu LC, Lopez-Vergès S, Barbour JD, Jones RB, Jha AR, Long BR, et al. Tim-3 marks human natural killer cell maturation and suppresses cell-mediated cytotoxicity. Blood. 2012 Apr 19;119(16):3734-43.

46. Dao TN, Utturkar S, Atallah Lanman N, Matosevic S. TIM-3 Expression Is Downregulated on Human NK Cells in Response to Cancer Targets in Synergy with Activation. Cancers (Basel). 2020 Aug 26;12(9).

47. Wang R, Jaw JJ, Stutzman NC, Zou Z, Sun PD. Natural killer cell-produced IFN-y and TNF-a induce target cell cytolysis through up-regulation of ICAM-1. Journal of Leukocyte Biology. 2012 2012/02/01;91(2):299-309.

48. Takeda K, Smyth MJ, Cretney E, Hayakawa Y, Kayagaki N, Yagita H, et al. Critical role for tumor necrosis factor-related apoptosis-inducing ligand in immune surveillance against tumor development. J Exp Med. 2002 Jan 21;195(2):161-9.

49. Kavanagh ME, Conroy MJ, Clarke NE, Gilmartin NT, O'Sullivan KE, Feighery R, et al. Impact of the inflammatory microenvironment on T-cell phenotype in the progression from reflux oesophagitis to Barrett oesophagus and oesophageal adenocarcinoma. Cancer Lett. 2016 Jan 1;370(1):117-24.

\section{Tables}

Table 1. Patient Demographic Table 


\begin{tabular}{|ll|}
\hline Age (years) (range) & 65.2 (48-83) \\
\hline Sex Ratio (M:F) & $22: 8$ \\
\hline Tumour Stage $^{1}$ & \\
\hline T0 & 5 \\
\hline T1 & 5 \\
\hline T2 & 3 \\
\hline T3 & 12 \\
\hline T4 & 3 \\
\hline Nodal Status & \\
\hline Positive & \\
\hline Negative & 16 \\
\hline Neo-Adjuvant Chemotherapy Regimen & 12 \\
\hline FLOT & 15 \\
\hline CROSS & 7 \\
\hline MAGIC & 1 \\
\hline FOLFOX & 1 \\
\hline Tumour Regression Grade & \\
\hline 1-2 & 135.38 \\
\hline 3-5 & $55 \%$ \\
\hline Mean VFA (cm $\left.{ }^{2}\right)^{3}$ & \\
\hline Viscerally Obese by VFA & \\
\hline
\end{tabular}

Figures 

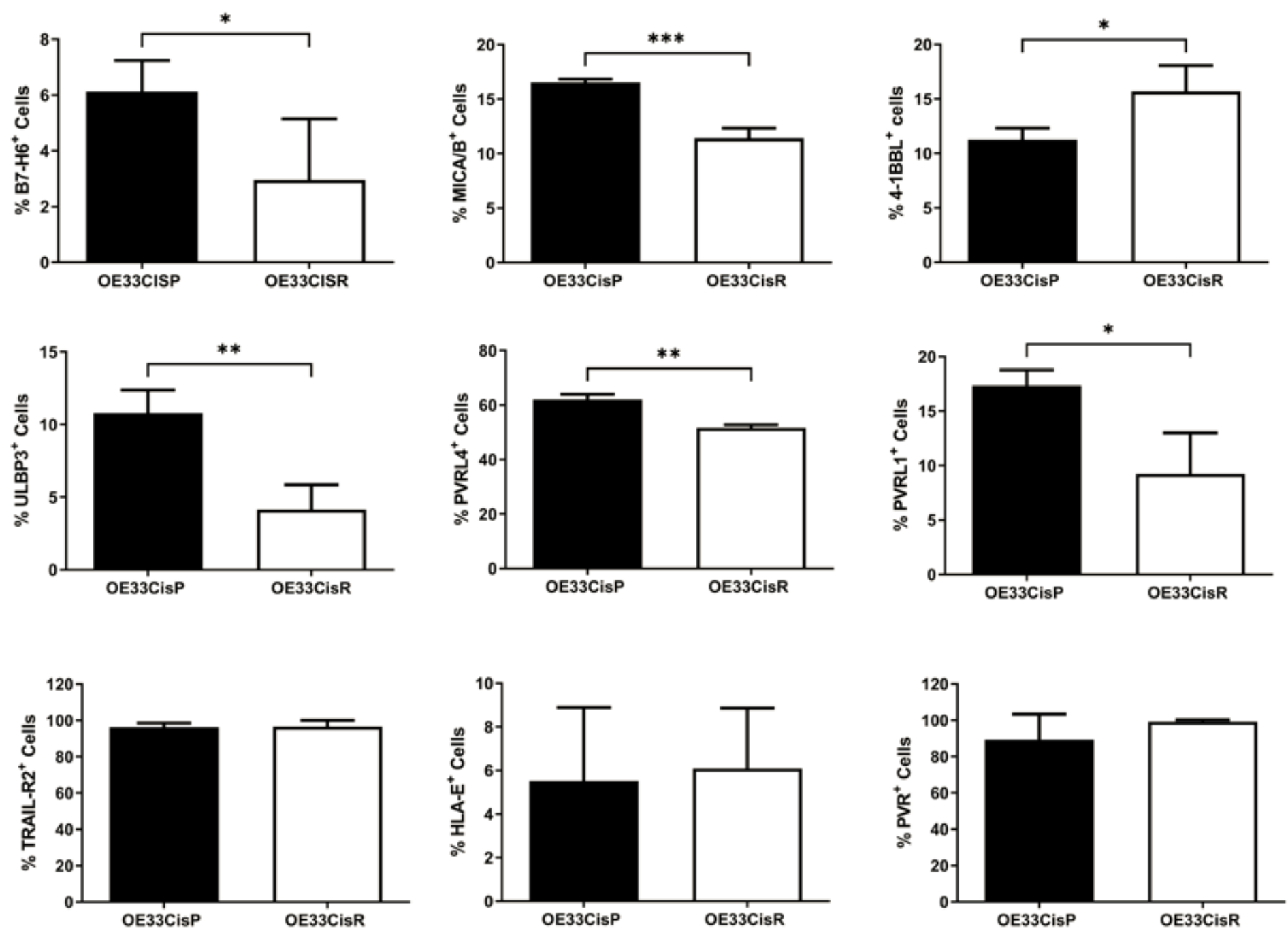

Figure 1

Significantly lower frequencies of OE33CisR cells expressing B7-H6, MICA/B, ULBP-3, PVRL-4 and PVRL-1 on their surface compared to OE33CisP cells. Bar chart showing the frequencies of OE33CisP and OE33CisR ( $n=3-5$ ) expressing NKR ligands B7-H6, MICA/B, 4-1BBL, ULBP-3, PVRL-4, PVRL-1, TRAIL-R2, HLA-E and PVR. ${ }^{*} p<0.05,{ }^{\star \star *} \mathrm{p}<0.001$ by unpaired t-test. [Mean+SEM]. 
B7-H6

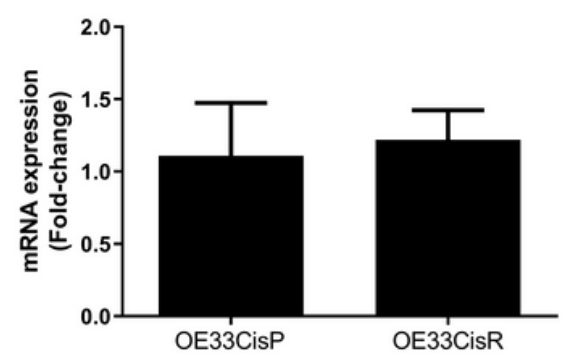

PVRL-4

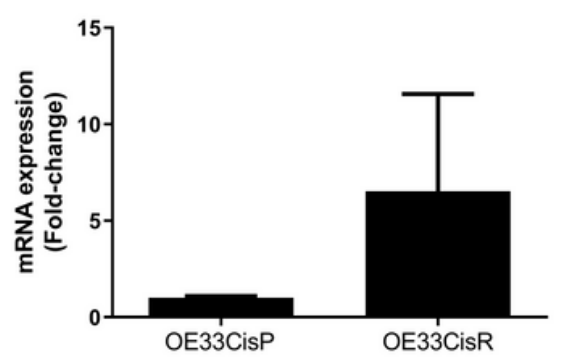

HLA-E

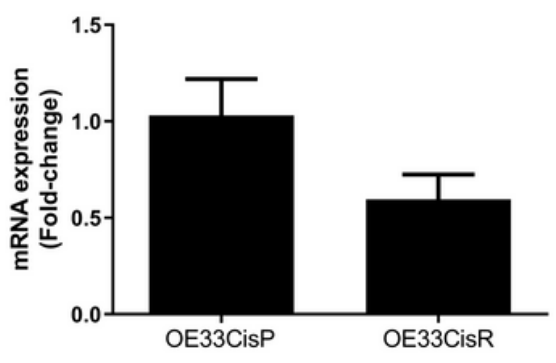

ULBP3

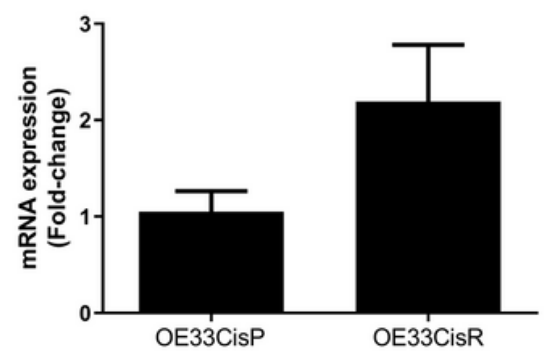

PVRL-1

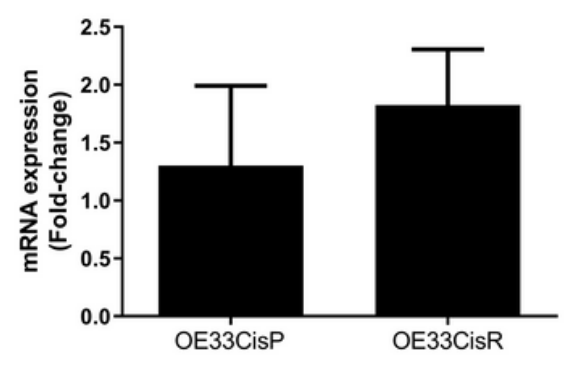

Figure 2

No significant differences in NK cell ligand mRNA expression between OE33CisP and OE33CisR cells. Bar chart showing the mRNA expression as fold change of the NK cell ligands (A) B7-H6, (B) HLA-E, (C) PVRL1, (D) PVRL-4 and (E) ULBP3 in OE33CisP (left) and OE33CisR (right). Gene expression for each gene is normalised to the housekeeping gene, $\beta$-actin. Unpaired t-test [Mean+SEM]. 
A

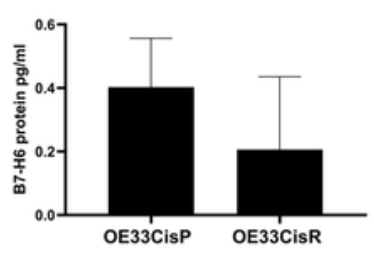

C

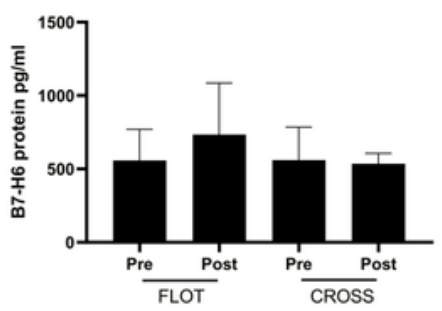

B

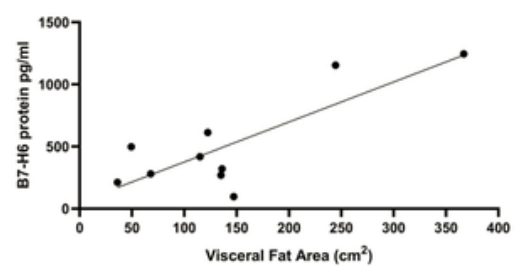

D

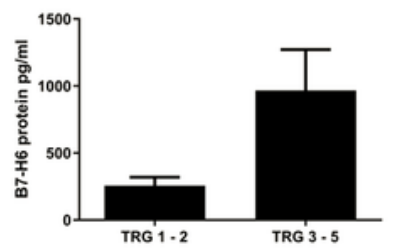

E

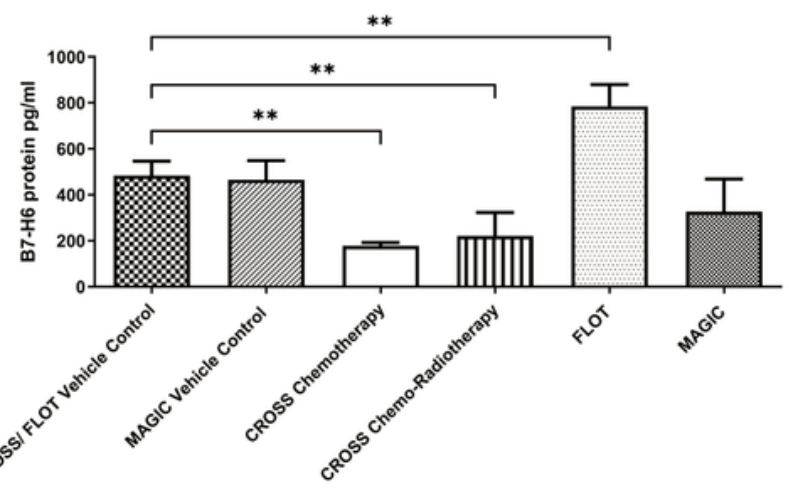

F

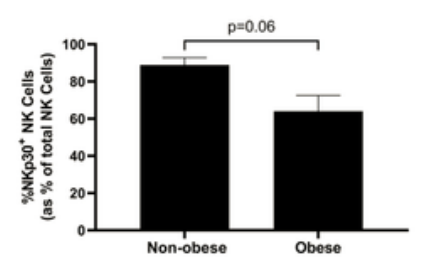

G

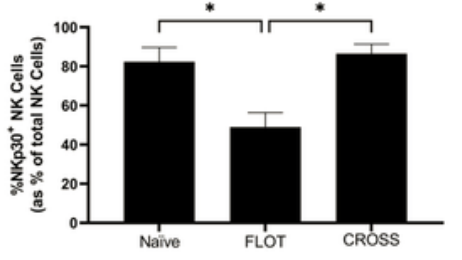

$\mathrm{H}$

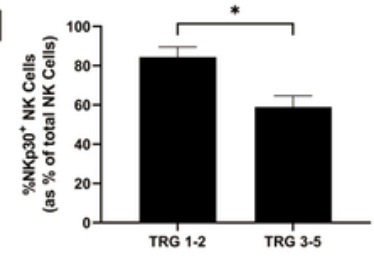

I

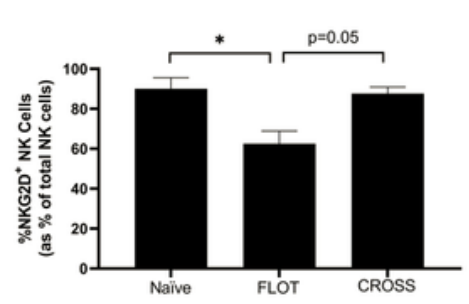

J

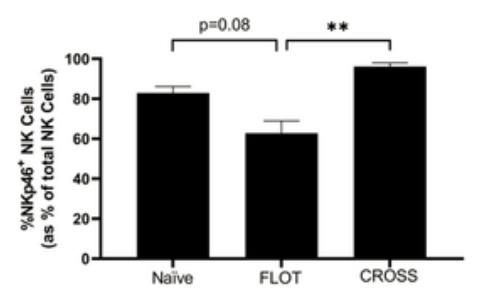

Figure 3

Differential levels of B7-H6 shedding and NKp30 surface expression in OAC patients following first-line treatments. (A) Bar chart showing the concentration of B7-H6 in OE33CisP and OE33CisR ( $n=3)$ cell supernatant, normalised to protein content $(\mu \mathrm{g} / \mathrm{ml})(B)$ Line graph showing correlation between visceral fat area (VFA) and B7-H6 protein levels in serum of 10 OAC patients. (C) Bar chart showing B7-H6 concentration in the serum of OAC patients pre-FLOT $(n=10)(0.8249 \mathrm{M}$ 5-fluorouracil, $2 \mu \mathrm{M}$ oxaliplatin, 
$0.001 \mu \mathrm{M}$ docetaxel) and post-FLOT $(n=5)(B L A C K)$ and pre-CROSS $(n=4)(0.001 \mu \mathrm{M}$ paclitaxel, $1000 \mu \mathrm{M}$ carboplatin, 2Gy irradiation) and post-CROSS ( $n=2$ ) (WHITE). (D) Bar chart showing B7-H6 concentration in the serum of OAC patients pre-treatment, stratified by tumour regression grade TRG 1-2 ( $n=4$, good responders) (BLACK) and TRG 3-5 ( $n=5$, poor responders) (WHITE) $n=5)$. (E) Bar chart showing the concentration of $\mathrm{B} 7-\mathrm{H} 6(\mathrm{pg} / \mathrm{ml})$ in OE33 cell supernatant following treatment with vehicle control, CROSS chemotherapy only, CROSS chemoradiotherapy (CRT), FLOT chemotherapies, or MAGIC chemotherapy (all n=3). FLOT (0.8249M 5-fluorouracil, $2 \mu \mathrm{M}$ oxaliplatin, $0.001 \mu \mathrm{M}$ docetaxel), CROSS $(0.001 \mu \mathrm{M}$ paclitaxel, $1000 \mu \mathrm{M}$ carboplatin, $2 \mathrm{~Gy}$ irradiation), MAGIC $(1.493 \mu \mathrm{M}$ epirubicin, $1.5 \mu \mathrm{M}$ cisplatin, $0.8249 \mu \mathrm{M}$ 5-fluorouracil. $(F)$ Bar chart showing the frequencies of ${\mathrm{NKp} 30^{+}}^{+} \mathrm{NK}$ cells in the circulation of non-obese $(n=5)$ and obese $(n=10)$ OAC patients by VFA. (G) Bar chart showing the frequency of circulating NKp30 NK cells following treatment FLOT $(n=9)$, CROSS $(n=4)$ or no treatment $(n=4)$ (treatment naïve) in OAC patients at time of surgical resection. $(\mathrm{H})$ Bar chart showing the showing the frequencies of $\mathrm{NKp} 30^{+} \mathrm{NK}$ cells in the circulation of OAC patients stratified by tumour regression grade TRG 1-2 ( $n=5$, good responders) and TRG 3-5 ( $n=5$, poor responders). (I) Bar chart showing the frequency of circulating NKG2D $D^{+}$NK cells following treatment FLOT $(n=10)$, CROSS $(n=4)$ or no treatment $(n=4)$ (treatment naïve) in OAC patients at time of surgical resection. $(\mathrm{J})$ Bar chart showing the frequency of circulating NKp46 ${ }^{+}$ NK cells following treatment FLOT $(n=9)$, CROSS $(n=4)$ or no treatment $(n=4)$ (treatment naïve) in OAC patients at time of surgical resection. ${ }^{*} p<0.05,{ }^{* *} p<0.01$ by One-way ANOVA with post-hoc Dunnett's or Tukey's test or t-test as appropriate. [Mean+SEM]. 
A
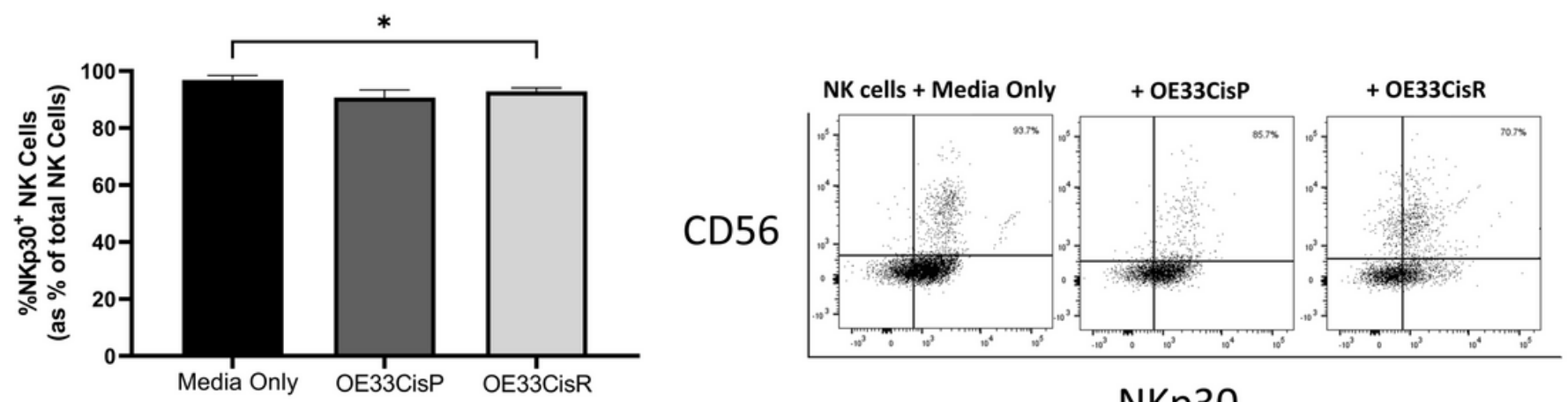

NKp30
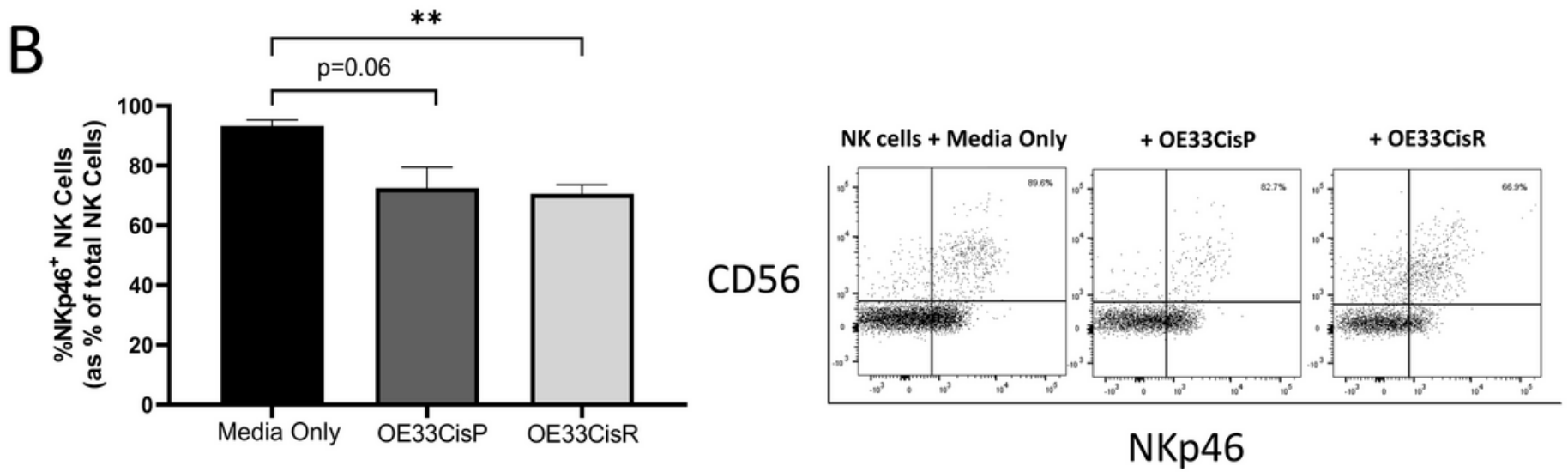

C
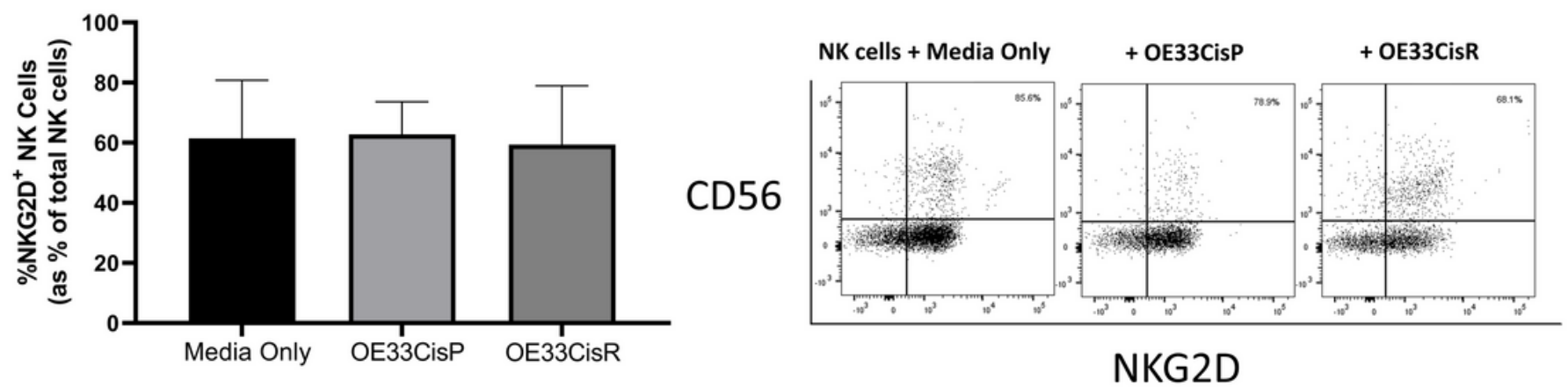

Figure 4

Significantly lower frequencies of NK cells expressing activating receptors following culture with cisplatin-resistant oesophageal cancer cells. (Left) Bar chart showing the frequencies of (A) NKp30 $\mathrm{NKp} 46^{+}$and (C) NKG2D $\mathrm{D}^{+}$NK cells following media only or co-culture with OE33CisP or OE33CisR cells (n=3-5). (Right) Representative dot plots showing (A) NKp30+, (B) NKp46 and (C) NKG2 ${ }^{+} \mathrm{NK}^{+}$cells previously gated on total lymphocytes Percentage frequencies shown are those of (A) NKp30, (B) NKp $46^{+}$and (C) NKG2D ${ }^{+}$NK cells as percentage of total NK cells. ${ }^{*} p<0.05,{ }^{*} p<0.01$ by one-way ANOVA with post-hoc Dunnett's test. [Mean+SEM]. 

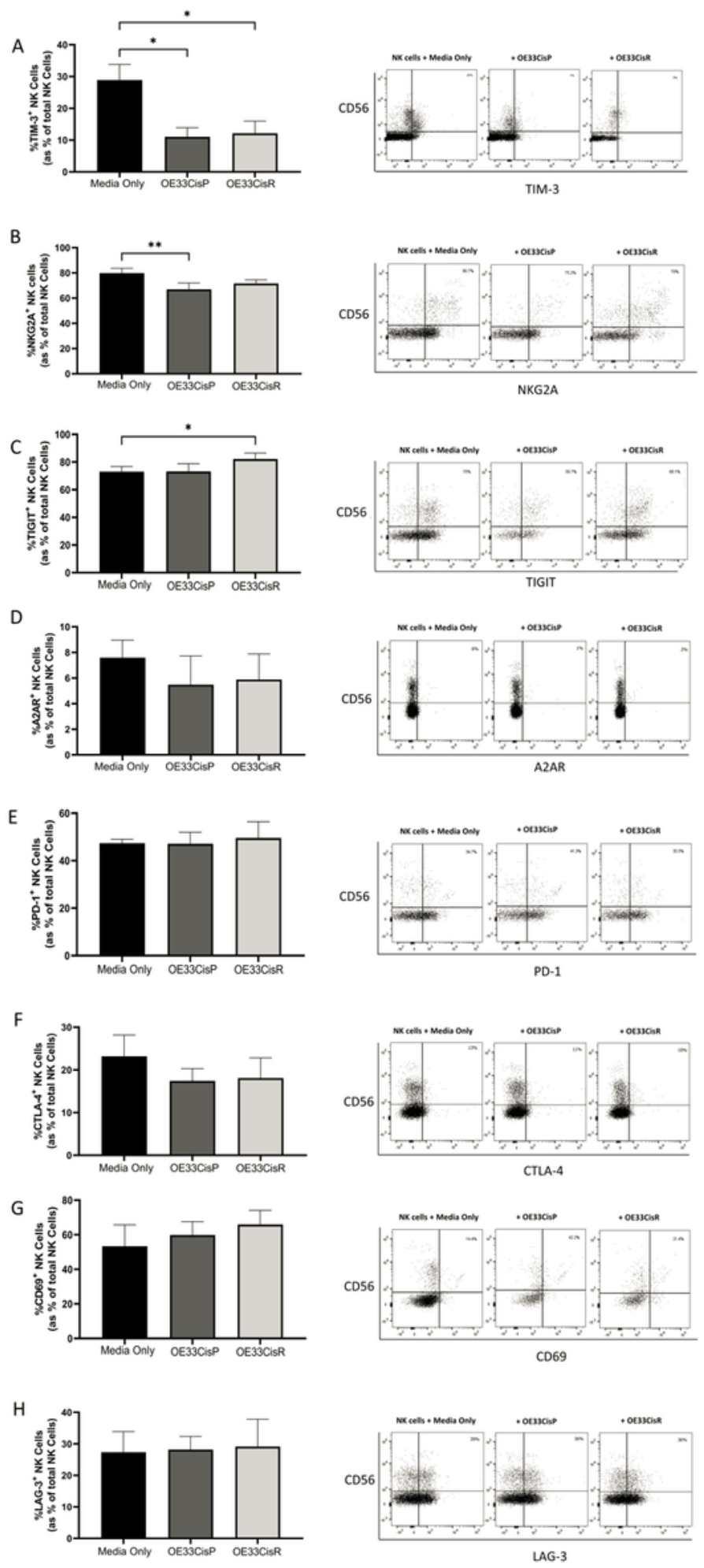

Figure 5

Significantly lower frequencies of TIM- $3^{+}$NK cells following co-culture with OE33Cisp or OE33CisR cells while frequencies of $\mathrm{TIGIT}^{+} \mathrm{NK}$ cells are higher following culture with OE33CisR. (Left) Bar chart showing

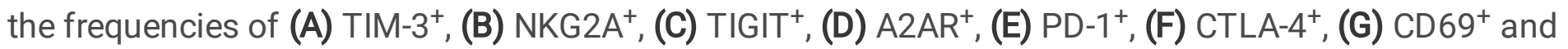
$(\mathrm{H}) \mathrm{LAG}-3^{+}$NK cells following culture alone or co-culture with OE33CisP or OE33CisR cells $(n=3)$. (Right) 


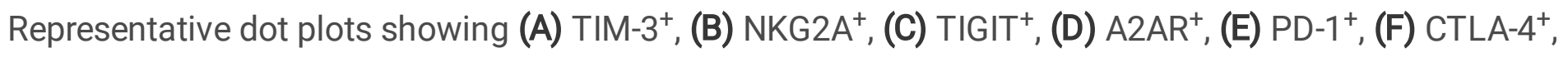
(G) $\mathrm{CD} 9^{+}$and $(\mathrm{H}) \mathrm{LAG}-3^{+} \mathrm{NK}$ cells previously gated on total lymphocytes Percentage frequencies shown

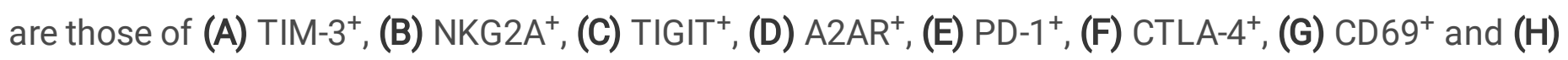
LAG- $3^{+}$NK cells as percentage of total NK cells. ${ }^{*} p<0.05$, ${ }^{* *} p<0.01$ by one-way ANOVA with post-hoc Dunnett's test. [Mean+SEM].
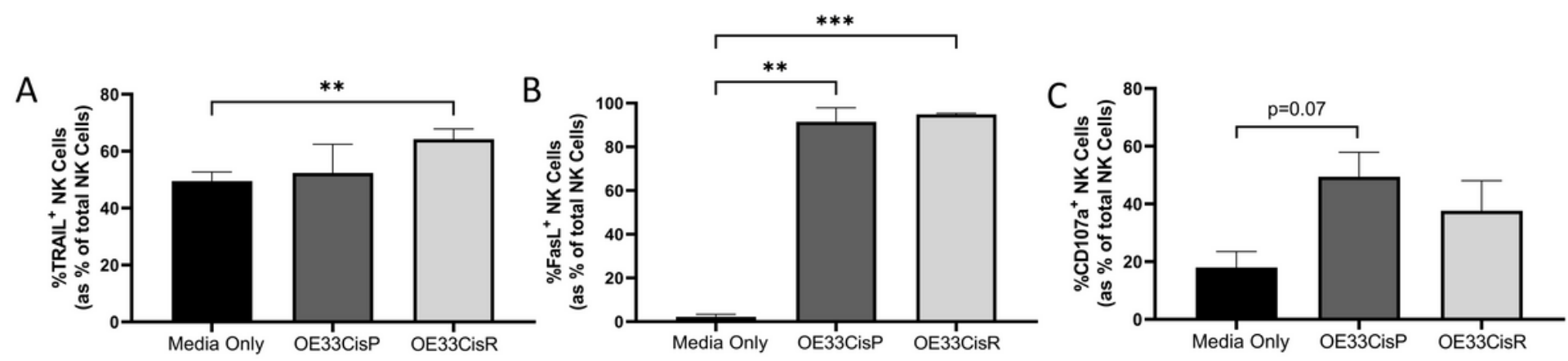

Figure 6

Significantly higher frequencies of NK cells expressing death receptor ligands following co-culture with cisplatin-resistant OE33 cells. Bar chart showing the frequencies of (A) FasL ${ }^{+}$, (B) TRAIL ${ }^{+}$and (C) CD107a ${ }^{+}$NK cells following culture alone or co-culture with OE33CisP or OE33CisR cells $(n=3) .{ }^{* *} p<0.01$, ${ }^{* \star *} \mathrm{p}<0.001$ by one-way ANOVA with post-hoc Dunnett's test. [Mean+SEM]. 

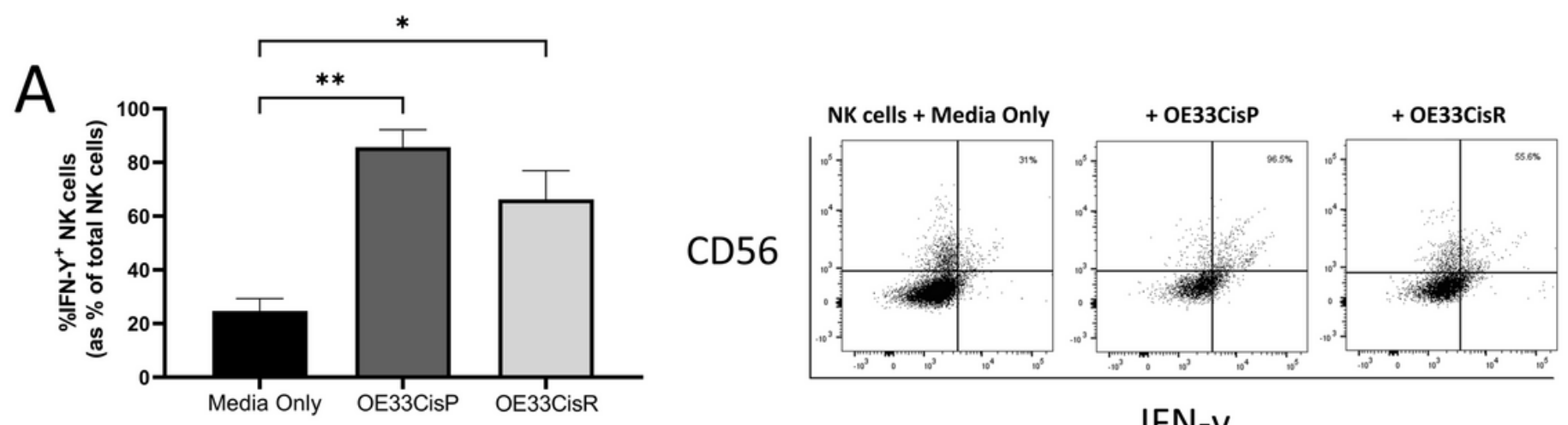

IFN-y
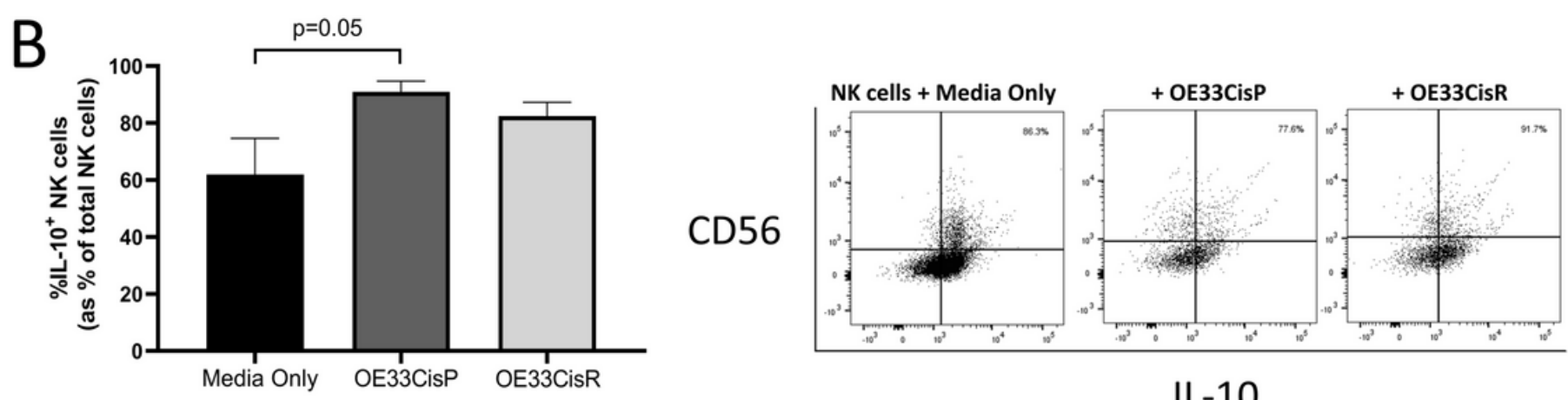

IL-10
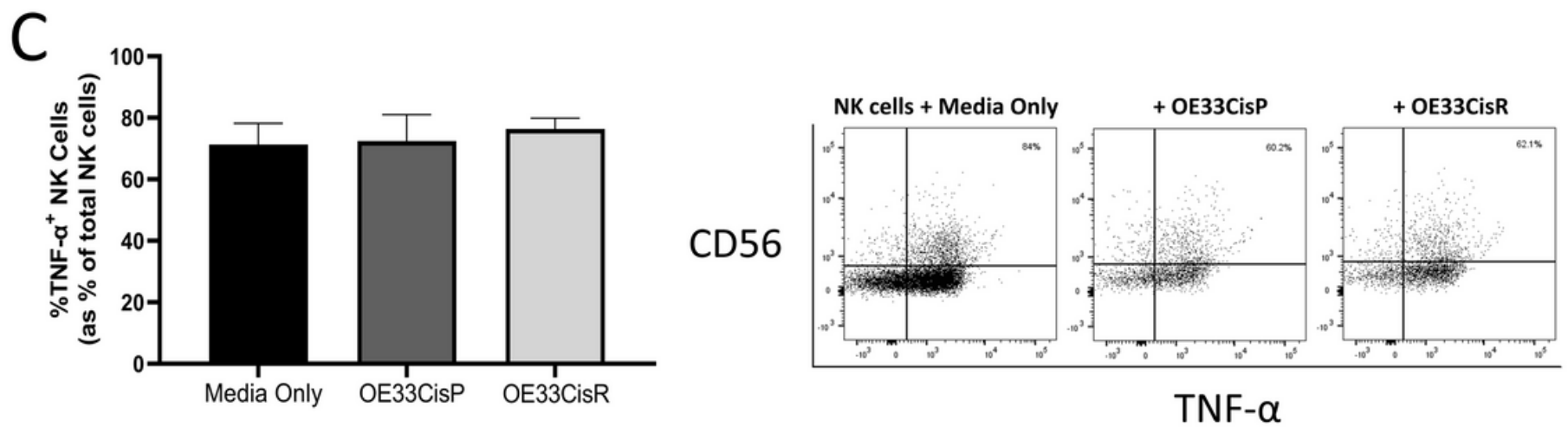

Figure 7

Significantly higher frequencies of NK cells producing IFN-y following co-culture with OAC cells. (Left) Bar chart showing the frequencies of (A) IFN- $\mathrm{Y}^{+},(\mathrm{B}) \mathrm{IL}-10^{+}$and (C) TNF-a ${ }^{+} \mathrm{NK}$ cells following culture alone or co-culture with OE33CisP or OE33CisR cells $(n=3)$. (Right) Representative dot plots for (A) IFN- $\mathrm{\gamma}^{+},(\mathrm{B}) \mathrm{IL}-$ $10^{+}$and (C) TNF-a ${ }^{+}$NK cells previously gated on total lymphocytes Percentage frequencies shown are those of (A) IFN- $\mathrm{Y}^{+}$, (B) IL-10 ${ }^{+}$and (C) TNF- $\mathrm{a}^{+} \mathrm{NK}$ cells as percentage of total NK cells. ${ }^{*} \mathrm{p}<0.05,{ }^{* *} \mathrm{p}<0.01$ by one-way ANOVA with post-hoc Dunnett's test. [Mean+SEM]. 